\title{
Subjective Quality Assessment of Longer Duration Video Sequences Delivered over HTTP Adaptive Streaming to Tablet Devices
}

\author{
Nicolas Staelens, Member, IEEE, Jonas De Meulenaere, Maxim Claeys, Student Member, IEEE, \\ Glenn Van Wallendael, Member, IEEE, Wendy Van den Broeck, Jan De Cock, Member, IEEE, \\ Rik Van de Walle, Member, IEEE, Piet Demeester, Fellow, IEEE and Filip De Turck, Senior Member, IEEE,
}

\begin{abstract}
HTTP Adaptive Streaming facilitates video streaming to mobile devices connected through heterogeneous networks without the need for a dedicated streaming infrastructure. By splitting different encoded versions of the same video into small segments, clients can continuously decide which segments to download based on available network resources and device characteristics. These encoded versions can, for example, differ in terms of bitrate and spatial or temporal resolution. However, as a result of dynamically selecting video segments, perceived video quality can fluctuate during playback which will impact end-users' Quality of Experience. Subjective studies have already been conducted to assess the influence of video delivery using HTTP Adaptive Streaming to mobile devices. Nevertheless, existing studies are limited to the evaluation of short video sequences in controlled environments. Research has already shown that video duration and assessment environment influence quality perception. Therefore, in this article, we go beyond the traditional ways for subjective quality evaluation by conducting novel experiments on tablet devices in more ecologically valid testing environments using longer duration video sequences. As such, we want to mimic realistic viewing behaviour as much as possible. Our results show that both video content and the range of quality switches significantly influence end-users' rating behaviour. In general, quality level switches are only perceived in high motion sequences or in case switching occurs between high and low quality video segments. Moreover, we also found that video stallings should be avoided during playback at all times.
\end{abstract}

Index Terms-Quality of Experience (QoE), Subjective video quality assessment, HTTP adaptive streaming, Mobile video, Tablet devices.

\section{INTRODUCTION}

$\mathbf{M}$ OBILE video consumption is growing at fast pace. According to a recent Internet traffic forecast report [1], $70 \%$ of the total mobile data traffic will be mobile video by 2016. At the same time, usage statistics [2] show that watching longer duration online videos, such as live streamed news, sports, and entertainment, is becoming more popular amongst viewers.

N. Staelens, M. Claeys, P. Demeester, and F. De Turck are with Ghent University - iMinds, Department of Information Technology, Ghent, Belgium (e-mail: \{nicolas.staelens, maxim.claeys, filip.deturck, piet.demeester\}@intec.ugent.be).

J. De Meulenaere and W. Van den Broeck are with the Vrije Universiteit Brussel - iMinds, Studies on Media, Information and Telecommunication, Brussels, Belgium (e-mail: \{jdemeule, wvdbroec\} @ vub.ac.be).

G. Van Wallendael, J. De Cock and R. Van de Walle are with Ghent University - iMinds, Department of Electronics and Information Systems, Ghent, Belgium (e-mail: \{glenn.vanwallendael, jan.decock, rik.vandewalle\}@ugent.be).
Mobile devices come in all different shapes and sizes, each with their specific characteristics and capabilities. Provisioning video to this wide variety of end devices connected through heterogeneous networks poses a challenge for online video service providers. Especially when these video services are provided Over-The-Top (OTT), i.e. delivered over the traditional packet-based best-effort Internet, network impairments and fluctuations in mobile bandwidth can deteriorate the (audio)visual quality of the video. In turn, this impacts endusers' Quality of Experience (QoE) [3] and appreciation of the offered video service. In this respect, ensuring and maintaining adequate QoE towards the end-users is of primary importance for video service providers [4].

HTTP Adaptive Streaming (HAS) [5] enables live and ondemand video streaming under varying network conditions to a wide range of end-devices, ranging from mobile phones to High Definition (HD) television sets. HAS operates by splitting the video into multiple smaller segments and encoding them at different quality levels (bitrates). While streaming video, HAS clients can continuously select which segments to receive based on device and network characteristics. For example, in case of bandwidth reduction, HAS clients can request segments of a lower quality level in order to ensure playback fluidity [6] and maintain QoE.

The advantages of HAS are that no special streaming server infrastructure is needed and that it runs natively over HTTP. The former implies a reduction of the deployment costs whereas the latter enables reliable content delivery over heterogeneous networks and automatically avoids firewall issues ${ }^{1}$. As HAS is delivered over reliable transport, network impairments such as packet loss result in an automatic retransmission of the lost data.

Nevertheless, due to the fact that HAS clients can dynamically adapt the video quality by selecting different segments, end-users' perceived visual quality is impacted [7]. Furthermore, since playback of each segment only starts when the segment has completely been received error-free, video freezes or stallings can also appear while watching the video [8].

Some research has already been conducted to investigate quality perception in the case of video delivery to mobile devices over HAS. Until now, these studies have been limited

\footnotetext{
${ }^{1}$ HAS traffic is identified as regular HTTP traffic, e.g. web browsing, and
} will therefore not be blocked by firewalls. 
to the evaluation of short duration video sequences $(10 \sim$ 15 seconds) or to quality assessment in stringent controlled environments, both as recommended by internationally standardized subjective quality assessment methodologies [9], [10]. However, subjective studies have also shown that content duration [11] and assessment environment [6] impact endusers' QoE.

In this article we are interested in assessing end-users' reactions to quality fluctuations when delivering video to tablet devices using HTTP Adaptive Streaming technologies. The research presented in this article goes a step further compared to the more traditional subjective video quality experiments by analysing the influence of longer duration audiovisual content and by conducting experiments in more ecologically valid testing environments. In this case, we assess video quality in subjects' home environment in order to mimic realistic viewing conditions as much as possible. The results obtained during this research provide insights into quality perception with respect to mobile video consumption under varying network conditions.

The remainder of this article is structured as follows. We start by providing an overview of the principles behind HTTP Adaptive Streaming and current existing standards in Section II. Then, in Section III, related work is presented with respect to subjective experiments assessing the influence of visual impairments during video delivery over HAS to mobile devices. In Section IV, we describe the approach followed to set up our new subjective video quality experiment in order to assess the influence of delivering longer duration video sequences using HAS to tablet devices. This includes a description of the selected video sequences, the simulation setup, and the real-life assessment environment. The results of this subjective experiment are analysed in Section V. Finally, the article is concluded in Section VI

\section{HTTP AdAPTIVE STREAMING}

Over the past decades, OTT video delivery has gained a lot of popularity, with HTTP Adaptive Streaming (HAS) becoming the de facto streaming technology. The general HAS concept is shown in Fig. 1. In HAS, a video is segmented into chunks with a typical length of $2 \mathrm{~s}$ to $10 \mathrm{~s}$. Furthermore, each of the segments are encoded at multiple quality levels. Information about the segments and quality levels is contained in a manifest file. At the client side, this manifest file can be used to link the different segments into a single video stream. Based on the information in the manifest file, a HAS client requests the segments in a progressive manner, while a buffer at the client side is used to take care of temporary anomalies such as a late arrival of a segment. Finally, the video segments are played back from the buffer as a continuous video stream.

Their OTT nature makes HAS techniques prone to bandwidth fluctuations and network congestion. This can introduce multiple types of visual impairments during video playout. Next to startup delay and playout interruptions caused by buffer starvations, bitrate switches also impact the end-users' perception of the video quality. Therefore, the HAS clients' intelligence in terms of quality selection is a crucial factor for

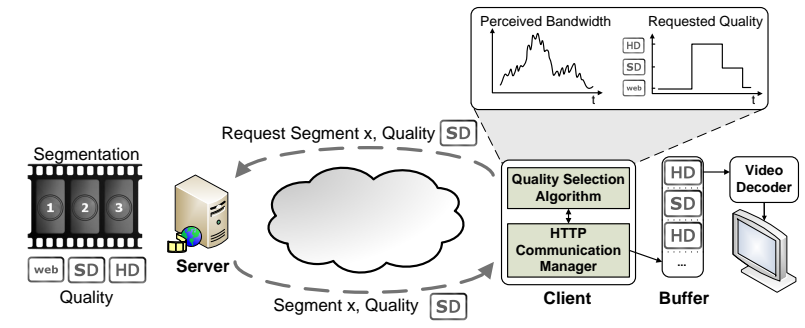

Fig. 1. Schematic overview of the HTTP Adaptive Streaming (HAS) concept.

the success of HAS techniques. A quality selection heuristic is applied to dynamically adapt the requested quality level in order to optimize the QoE, based on the network conditions, perceived while downloading previous segments.

Several large industrial players have commercial implementations of the HAS concept. These implementations include, amongst others, Microsoft IIS Smooth Streaming (MSS) ${ }^{2}$, Apple's HTTP Live Streaming (HLS) ${ }^{3}$ and HTTP Dynamic Streaming ${ }^{4}$ by Adobe. Next to the commercial implementations, multiple quality selection heuristics have been proposed in academia. While most of these approaches are deterministic [12], [13], [14], recently, self-learning HAS clients have been proposed, claiming to outperform deterministic approaches in variable network conditions [15], [16]. The performance of these self-learning HAS clients heavily depends on the accuracy of the underlying model of the end-users' QoE.

In 2011, MPEG tried to find the common ground between the variety of HAS implementations with Dynamic Adaptive Streaming over HTTP (DASH) [5], [17]. In DASH, only the interfaces and protocol data of the HAS concept are standardized. The quality selection heuristics are, however, not standardized and thus depend on the specific implementation.

\section{OVERVIEW OF SUBJECTIVE VIDEO QUALITY EXPERIMENTS ON MOBILE DEVICES}

A number of studies have already been performed to assess the influence of visual impairments during video delivery over HTTP Adaptive Streaming technologies. This includes studying the effects of startup delay, playout interruptions, and bitrate switches on perceived video quality as well as conducting subjective experiments on mobile devices (e.g., smartphones, tablets, ....).

In [18], the authors investigated how perceived quality is influenced by video encoding, video content, and display device. Several video sequences were evaluated by a test panel on a TV display, tablet device, and smartphone. Results show that perceived quality is indeed impacted by the display device and that subjects may have higher quality expectations for devices with a larger screen. Catellier et al. [19] also assessed the impact of different mobile devices, ranging from a smartphone to a laptop, on perceived multimedia quality. They found that the influence of the device and screen size has no significant

\footnotetext{
${ }^{2}$ http://www.iis.net/downloads/microsoft/smooth-streaming

${ }^{3}$ http://tools.ietf.org/html/draft-pantos-http-live-streaming-10

${ }^{4}$ http://www.adobe.com/products/hds-dynamic-streaming.html
} 
influence on quality perception in the case of watching highquality video sequences. In the case of medium and lowquality sequences, differences can be observed between mobile devices and devices with larger screens (e.g. television sets). Moreover, depending on the experience with online and mobile video, users are more tolerant towards the occurrence of visual impairments during video playback [20].

As part of the LIVE Mobile Video Quality Assessment database [7], subjective experiments were conducted to assess the influence of temporally varying compression rates and frame freezes on quality perception. Short video sequences (15 seconds duration) with a resolution of $1280 \times 720$ pixels were used for conducting the subjective experiments on a smartphone and a tablet device. No differences were found between the smartphone and the tablet users in terms of quality perception and evaluation, and results indicate that

- longer frame freezes are preferred to multiple short freezes,

- multiple rate switches are preferred over fewer if, as a result, subjects can watch the high quality video for a longer duration,

- switching to an intermediate rate before switching to a higher rate is preferred over multiple large magnitude rate switches similar to the results in [21],

- and that a continuous rate is preferred over switches if the continuous rate is higher than the base rate over the switches.

Avoiding large magnitude rate switches is supported by the Weber-Fechner law [22] which states that the just noticeable difference between the current and the previous stimuli is proportional to the magnitude of the stimuli. For example, results in [23] and [24] show that the impact of quality fluctuations during quality assessment is relative to the current perceived quality level. The fact that frequent bitrate switches should be avoided was also concluded based on the experiments conducted in [25]. Results show that a nearly constant quality is preferred over an oscillating one and that content genre impacts impairment visibility. In this case, two different video sequences containing several visual artefacts typical for HTTP adaptive streaming were displayed on a PC to a number of test subjects.

Recently, Floris et al. [26] and Atzori et al. [27] performed subjective quality evaluations on different tablet devices using low resolution (Common Intermediate Format; 352×288 pixels) video sequences. The authors consider the influence of startup delay, buffer starvations, and bitrate switches on perceived video quality. Interestingly, the perceived quality depends on the type of tablet used (iPad 2 or Galaxy Tab). It appears that test subjects have increased quality expectations when watching video on iPad tablets. Similar to the research findings in [7], [28], and [8], the authors also found that buffer starvations (resulting in freezes or stallings) should be avoided. As such, a higher startup delay is preferred over buffer starvations during video playout. Furthermore, depending on the specific type of application, users tolerate different amounts of initial startup delays [8]. Research in [6] shows that playback fluidity is especially important when watching television. In the case of video stallings, the typical lean backward TV viewing experience is hampered resulting in a disruption of subjects' immersive experience. Dobrian et al. [29] also showed that the number of buffer starvations has a direct negative impact on end-users' engagement. In [30], an analytical model is proposed to estimate the amount of initial buffering needed in order to keep the probability of playback interruptions below certain thresholds. Video playout interruption probabilities can be estimated using the analytical model proposed by Xu et al. [31].

All of the aforementioned subjective experiments have been conducted using short duration video sequences, except for the work from Robinson et al. [25]. This corresponds to the guidelines provided by internationally standardized subjective video quality assessment methodologies [9], [10]. However, research has shown that sequence duration impacts subjects' rating behaviour [6], [11], [32]. Also, as context of use influences quality perception [6], [33], subjective quality assessment experiments should be conducted in more ecologically valid testing environments (i.e. field tests).

\section{ASSESSING VISUAL IMPAIRMENTS IN LONG DURATION VIDEOS OVER HTTP ADAPTIVE STREAMING}

In this section, we detail the approach we followed [34] for setting up our subjective video quality assessment experiment. This includes an overview of the selected video sequences, the encoding parameters, and the toolchain for simulating video delivery over HAS. More information is also provided on the assessment methodology used for presenting the different video sequences to the test subjects and gathering quality rating.

\section{A. Source video sequence selection}

In order to conduct subjective video quality assessment experiments, a number of source video sequences (SRCs) must first be selected which are representative for the targeted use case [35]. The latter is important as content desirability, immersion, and content characteristics (such as amount of motion and spatial details) influence quality perception [6], [36], [37].

The amount of motion and spatial details in a video sequence can be quantified by means of the Spatial Information (SI) and Temporal Information (TI) indices as described in ITU-T Recommendation P.910 [9]. In this recommendation, SI and TI are calculated for each video frame and the maximum value over all frames is taken as overall value for the entire video sequence. However, in order to avoid the influence of peak values, Ostaszewska et al. [38] propose the use of the upper quartile value as overall SI and TI measurement.

As reported in [2], sports and entertainment are amongst the most watched content online. We selected six different SRCs with accompanying audio, taken from Blu-ray discs, to be used in this subjective experiment. These six sequences correspond to different content classes spanning a wide range of different content characteristics as shown in Table I. All sequences are in full High Definition resolution (1920x1080 pixels) at 24 frames per second (fps). 
TABLE I

SPATIAL AND TEMPORAL INFORMATION [38] FOR CONTENT TYPES USED IN THE SUBJECTIVE EXPERIMENT.

\begin{tabular}{|l|c|c|}
\hline Sequence & Q3.SI & Q3.TI \\
\hline \hline Action & 30,32 & 26,10 \\
Cartoon & 22,97 & 20,33 \\
Drama & 29,36 & 7,46 \\
Music & 27,04 & 14,05 \\
Nature & 44,86 & 10,42 \\
Sports & 81,18 & 32,52 \\
\hline
\end{tabular}

In the previous section, it was mentioned that content duration influences subjects' rating behaviour. Furthermore, the authors in [25] state that HAS protocols may receive lower quality ratings in the case of using short duration video sequences. Thus, similar to the experiment in [25], sequence duration was selected to be around two minutes. This enables us to assess the influence of multiple bitrate switches and frame freezes, and allows subjects to evaluate multiple videos within a single subjective experiment.

\section{B. Encoding for HTTP Adaptive Streaming}

For encoding different versions of the video sequences we followed Apple's Technical Note TN2224 specifications [39]. As such, we used realistic encoding settings for simulating video deployment over HAS. However, instead of using segments of 10 seconds, we employ segments with a two seconds duration. Smaller segments enable a faster startup time and faster rate adaptations in case of bandwidth fluctuations which is important for real-time video streaming [13], [40]. All sequences were encoded in H.264/AVC using $x 264$ based on the parameters listed in Table II.

TABLE II

PARAMETERS USED FOR ENCODING THE SOURCE VIDEO SEQUENCES FOR SIMULATING VIDEO DISTRIBUTION OVER HAS. THESE PARAMETERS ARE PARTIALLY TAKEN FROM [39].

\begin{tabular}{|c|c|c|c|}
\hline $\begin{array}{c}\text { Quality } \\
\text { level }\end{array}$ & $\begin{array}{c}\text { Resolution } \\
\text { (in pixels) }\end{array}$ & $\begin{array}{c}\text { Video Bitrate } \\
\text { (in kbps) }\end{array}$ & Profile \\
\hline \hline 1 & $640 \times 360$ & 600 & Baseline \\
2 & $640 \times 360$ & 1200 & Baseline \\
3 & $960 \times 540$ & 1800 & Main \\
4 & $960 \times 540$ & 2500 & Main \\
5 & $1280 \times 720$ & 4500 & Main \\
6 & $1280 \times 720$ & 6500 & Main \\
7 & $1920 \times 1080$ & 8500 & High \\
\hline
\end{tabular}

A keyframe interval of 48 was used in order to match the segment duration of 2 seconds. As such, each new segment starts with a keyframe. Advanced Audio Coding (AAC) was used for encoding the accompanying stereo audio track at a data rate of $64 \mathrm{kbit} / \mathrm{s}$ and a sampling rate of $44100 \mathrm{~Hz}$.

\section{Simulating video delivery over HTTP Adaptive Streaming}

In this experiment, we are interested in assessing the influence of quality fluctuations on end-users' QoE during video delivery over HAS. More specifically, we want to investigate end-users' reactions to bitrate switches and video stallings caused by buffer starvations during video playback. Furthermore, we also want to validate whether the results obtained using short video sequences in controlled environments (cfr. Section III) are still valid in the case of quality evaluation using longer duration video content in real-life environments.

Accordingly, three different scenarios with a fixed duration of 108 seconds were selected in order to evaluate

1) the impact of bitrate fluctuations on current quality perception [23], [24] and the influence of buffer starvations [26], [27],

2) the influence of frequent bitrate switches [25],

3 ) the difference between multi-ranges bitrate switches and gradual bitrate switches [7], [21],

on quality perception. These scenarios are also graphically represented in Fig. 2.

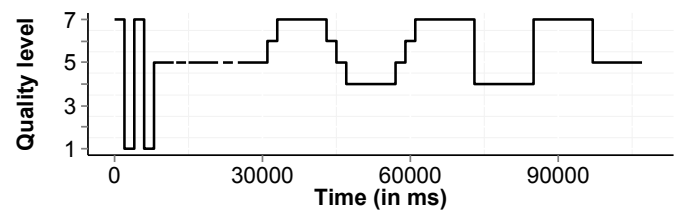

(a) Scenario 1

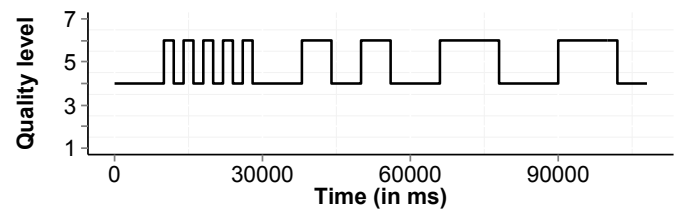

(b) Scenario 2

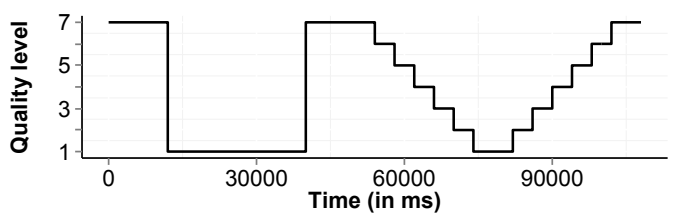

(c) Scenario 3

Fig. 2. Graphical overview of the three selected adaptive streaming scenarios to assess end-users' reactions to bitrate switches and video stallings. Each scenario has a fixed duration of 108 seconds. Gaps in graph (a) represent the occurrence of video stallings.

More detailed information on the durations of each quality switch, for each scenario, is provided in Table III. The duration of each quality level is indicated using the following notation: $<$ quality_level $>-<$ duration (in $s$ ) $>$;

In order to ensure that exactly the same impaired video sequences can be evaluated by different test subjects during our subjective video quality experiment, we opted to simulate the above mentioned scenarios rather than having a real streaming setup. The toolchain depicted Fig. 3 was used to simulate video delivery over HAS.

Each SRC is first encoded into seven different quality levels based on Apple's TN2224 specifications as detailed in the previous section. Each quality level corresponds to an encoded version of the original sequence at a specific resolution and bitrate (see Table II). Next, these quality levels and accompanying audio track are decoded, upscaled, and segmented into raw YUV 4:2:0 video segments of 2 seconds. Upscaling to 
TABLE III

DETAILED TIMING INFORMATION FOR EACH OF THE SCENARIOS. TUPLES OF THE FORM $<$ quality_level $>$-<duration (in $s$ ) $>$; INDICATE THE TIME A QUALITY LEVEL IS MAINTAINED AND THE TRANSITION FROM ONE QUALITY LEVEL TO ANOTHER.

Scenario 1

$7-2 ; 1-2 ; 7-2 ; 1-2 ;$

5-4;(freeze 0.5s);5-2;(freeze 0.5s);5-6;(freeze 1s);5-2;(freeze 1s);5-6; $6-2 ; 7-10 ; 6-2 ; 5-2 ; 4-10 ; 5-2 ; 6-2$;

$7-12 ; 4-12 ; 7-12 ; 5-11$;

Scenario 2

$4-10 ; 6-2 ; 4-2 ; 6-2 ; 4-2 ; 6-2 ; 4-2 ; 6-2 ; 4-2 ; 6-2$;

$4-10 ; 6-6 ; 4-6 ; 6-6$;

$4-10 ; 6-12 ; 4-12 ; 6-12 ; 4-6$;

Scenario 3

7-12;1-28;7-14;

6-4;5-4;4-4;3-4;2-4;1-8;2-4;3-4;4-4;5-4;6-4;7-6;

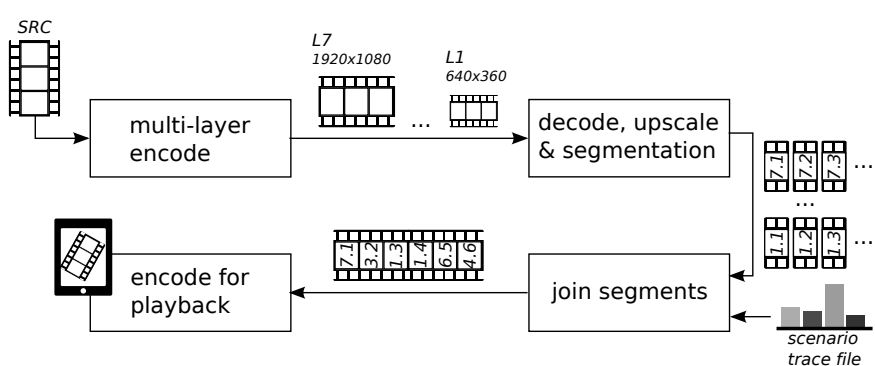

Fig. 3. Toolchain for simulating video delivery over HAS in order to ensure all subjects evaluate exactly the same impaired video sequences during our subjective experiment.

the highest video resolution is performed as we assume the video sequence is always played in fullscreen. As such, we simulate the upscaling which would otherwise be done by the device itself when playing each individual segment. For this step, we used Lanczos resampling. Finally, using trace files representing each of the three streaming scenarios listed above, the different segments from the different quality levels are concatenated to create one complete video sequence as it would appear to the subjects when streaming it over HAS. Video stallings are simulated by inserting frame freezes with silent audio in the video sequence. As the resulting video sequence is still in raw YUV 4:2:0 format, a final encoding step is required to make the sequences playable on tablet devices. The video part was again encoded using $x 264$. During this last encoding stage, a Constant Rate Factor (CRF) of 15 was used in order to ensure the absence of any additional encoding artefacts ${ }^{5}$. This was also confirmed visually. As explained in the previous section, the quality of the audio track remained unchanged while processing the video.

The resulting video sequences where all locally stored on the tablet device in order to ensure all subjects evaluated exactly the same videos during the experiment.

\footnotetext{
${ }^{5}$ This resulted in an average encoding PSNR of 50dB.
}

\section{Subjective quality assessment methodology and environ-} ment

Our subjective quality assessment is based on longer duration video sequences subject to time-varying quality fluctuations. Since we are interested in capturing end-users' reactions to these changes in perceived video quality, receiving instantaneous feedback is preferred. This also enables us to track the impact of previous quality levels on the current perceived quality.

Therefore, we selected the Single Stimulus Continuous Quality Evaluation (SSCQE) [10] subjective assessment methodology as base for conducting our experiment. Using this methodology, rapid changes in quality can be tracked [37], [41] and subjects can continuously adjust their quality rating during video playback. We have implemented this methodology to enable quality evaluation on tablet devices, more specifically fourth generation iPads. As depicted in Fig. 4, subjects were presented with a slider at the bottom of the screen to adjust their quality rating.

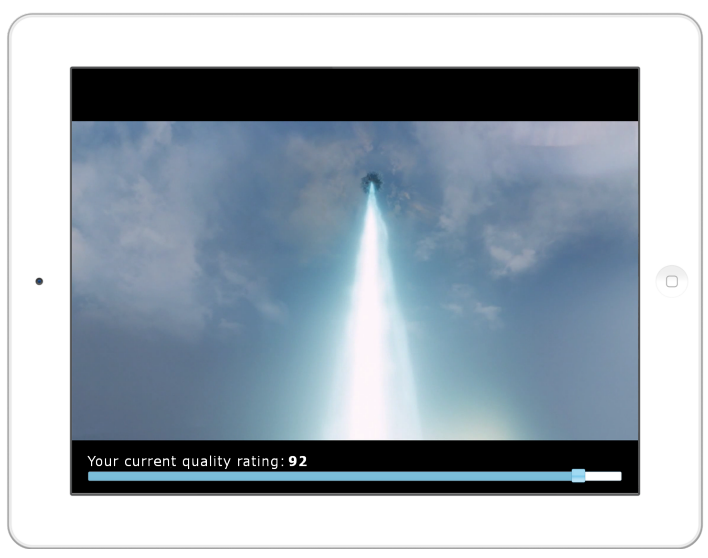

Fig. 4. Screenshot of the implemented SSCQE subjective assessment methodology. Using the slider, subjects can instantaneously adjust their quality rating.

Before the start of the experiment, subjects were informed on how to evaluate the different video sequences. Specific instructions were provided to emphasize the importance of continuously adjusting their quality rating using the slider while watching the video. The slider ranged from 0 to 100 , where 100 stands for 'perfect' quality. Also, one training sequence was used to get the subjects familiarized with the experiment. During the experiment, all video sequences (18 in total) were randomly shown one-after-another. Before the start of each sequence, we inserted a short pause of 5 seconds. Also, at the start of each sequence, the slider position was restored to the middle (50).

Traditionally, subjective quality assessment experiments are conducted in controlled environments [9], [10] with stringent requirements imposed on room lighting conditions, screen calibration, viewing distance, and subjects' seating position. However, research has also shown that assessing quality in more realistic environments can impact users' quality perception and overall QoE [6], [33], [42]. Therefore, in order to better understand QoE, Van den Broeck et al. [43] argue that 
the everyday-life context should be integrated in subjective video quality experiments. In the study presented in this article, the latter is achieved by actually going to the people's home and let them watch the video sequences on the tablet device in their typical home environment. As such, subjects are placed in a more ecologically valid testing environment.

\section{E. Test subjects}

A total number of 26 test subjects participated in our experiment, ten of which were females. The age of the female test subjects ranged from 24 to 31 years old with an average age of 28 . The male subjects were aged between 22 and 65 years old, with an average age of 32 .

Subjects were allowed to select their own most comfortable seating position and viewing distance as they would normally use the tablet device. On average, the viewing distance between the subject's eyes and the tablet's screen was around 15.32 inches with a standard deviation of 3.69 inches. This corresponds to a viewing distance close to three times the actual iPad's screen height (in landscape mode). Subjects did not change this seating position and viewing distance during the experiment.

\section{RESULTS}

As recommended by ITU-R Recommendation BT.500, the continuous raw data from each of the test subjects was first sampled every $500 \mathrm{~ms}$ and averaged over all test subjects before processing and analysing the data further.

In the case of continuous quality evaluation, users' (instantaneous) feedback can be slightly delayed due to the variation in user response times [10]. In other words, when a visual stimulus changes, subjects need some time to react to these changes and adjust their quality rating. Therefore, SSCQE scores are typically globally time-shifted in order to compensate for this delay in response times [44], [45], [46]. In our research, we are primarily interested in assessing end-users' reactions to quality fluctuations, not in estimating perceived video quality. Therefore, we do not compensate for shifts in response times.

In [47], the authors first convert the raw quality ratings to standardized and normalized Z-scores in order to compensate for differences in subjects' quality scale usage. In our case, converting the raw scores to $\mathrm{Z}$-scores reduced the deviation between test subjects without affecting the overall average rating behaviour. As such, for our data analysis, converting the data to Z-scores did not prove very useful.

In this work, we thus present the continuous quality ratings. The work of Seufert et al. [48] shows that the overall QoE of HTTP Adaptive video streams can be estimated with high accuracy by simply taking the mean values of the different quality levels.

\section{A. Scenario 1: Influence of bitrate fluctuations and video stallings}

As depicted in the upper part of Fig. 5, the first scenario contains a wide variety of bitrate switches, including multirange switches, gradual switches, and short and longer duration switches. Also, four rebuffering events are simulated resulting in two video stallings of 0.5 seconds in duration followed by two stalling of 1 second. These video stallings are represented by gaps in the scenario plot.
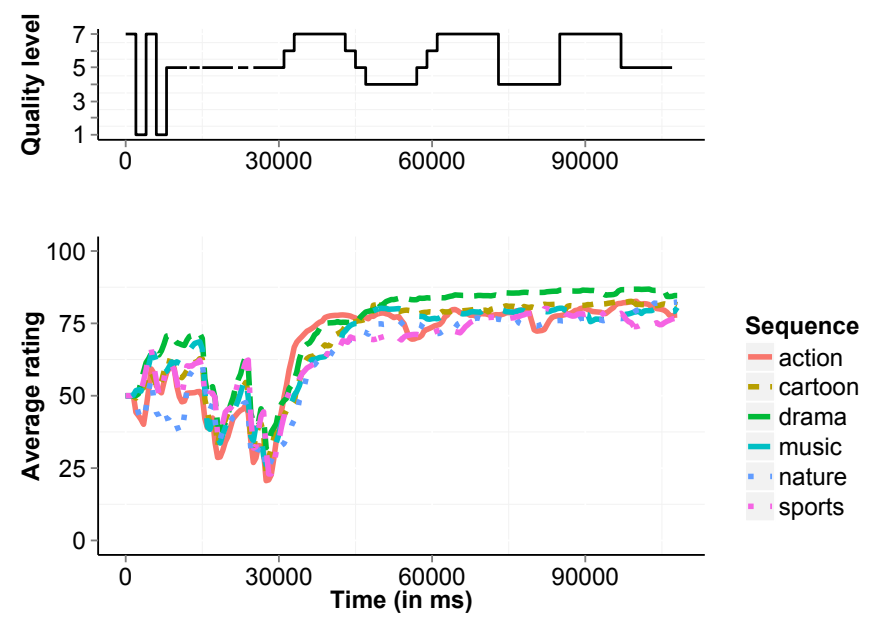

Fig. 5. Overview of the quality switches during video playback in order to assess the influence of bitrate fluctuations and video stallings; and corresponding average quality ratings per video sequence.

The average quality ratings for each of the content types are plotted in the lower part of Fig. 5. Using a Mann-Whitney U Test, we found that there are significant differences between the average quality ratings of the different sequences. Hence, in correspondence to the results in [18], content influences quality ratings and rating behaviour.

As shown in Table II, target bitrates were used to encode the quality levels of the video streams. When targeting a certain bitrate, the resulting encoding quality also depends on video characteristics. As such, easy-to-code sequences will have a higher visual quality compared to hard-to-code videos [49].

The results in Fig. 5 show that the test subjects are able to track the quality fluctuations during video playback. We found that the average delay in viewers' response times is around two seconds. The saturation effect [37], [50] causes subjects not to use the extremes of the rating scale. Hence, the highest quality level does not necessarily result in a quality rating of 100 .

The multi-range bitrate switches in the beginning of the sequence have a slightly higher impact on the quality ratings for the action, nature and sports sequence. This corresponds with the harder-to-code sequences which contain a lot of spatial details and texture (see Table I). In case of the drama sequence, which contains the least amount of motion, the bitrate fluctuations are not visible resulting in a nearly constant average quality rating.

From the graph it shows that the short multi-range bitrate switches are rated worse quality compared to longer duration mid-quality switches. No real difference can be observed between switching to a mid-quality levels gradually or instantaneously. As such, there are limited noticeable differences between the high and mid-quality levels of the video stream [22] which impact quality ratings [23], [24]. In the third scenario (Section V-C), we will investigate whether this is also the case when switching gradually or instantaneously between the highest and the lowest quality levels of the video stream. 
It is clear that the occurrence of frame freezes (video stallings) during playback have a high impact on the quality ratings. In the case of video stallings, a significant drop can be observed in the average quality ratings for all the video sequences. Additionally, consecutive stallings result each time in a lower appreciation of the video's QoE. This corresponds to our earlier research findings [6] that video stallings negatively influence viewers' immersive experience. As such, corresponding to the results in [7], [8], [28], buffer starvations resulting in video stallings should be avoided at all times during video playback.

\section{B. Scenario 2: Influence of frequent bitrate switches}

In order to assess the influence of the frequency of bitrate switches, we created a scenario with oscillating segment quality. In this case, the mid-range level switches differ both in frequency and duration as shown in Fig. 6.
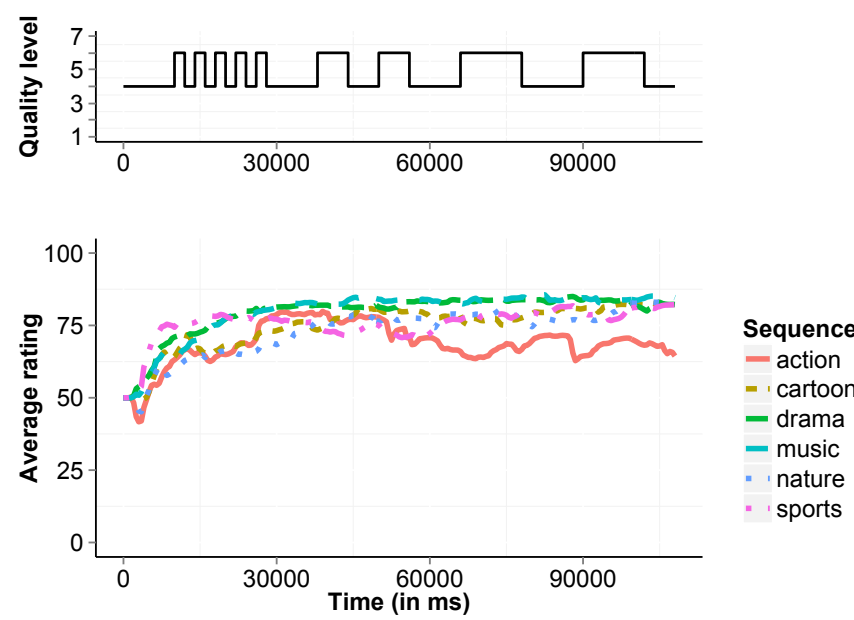

Fig. 6. Overview of the quality switches during video playback in order to assess the influence of frequent bitrate switches on quality perception; and corresponding average quality ratings per video sequence.

The average quality ratings in Fig. 6 indicate that the midrange quality level switches are in most cases not perceived by our test subjects. This is in line with our findings from the previous scenario that there are limited noticeable differences between the mid-range quality oscillations of our video sequences. Especially in the case of video sequences with low amounts of motion and spatial detail (drama and music), the mid-range quality fluctuations are actually not perceived at all.

In case of the nature and cartoon sequence, the frequent quality level switches at the beginning of the video playback result in a lower average quality rating compared to maintaining a lower quality level for a longer period in time as was also found in [7] and [23]. Less frequent switches have again no real impact on the perceived video quality.

With exception of the action sequence, the frequent midrange quality switches do not result in a decrease of the average perceived quality but yield a stabilisation of the ratings. In slight contrast, the frequent full-range quality switches from the previous scenario do result in a decrease of quality perception. Hence, not only the frequency of the switches impacts end-users' perceived quality but also the range between the quality levels during the switches.

The average rating behaviour for the action sequence deviates the most from the other video sequences. This scene is characterized by high motion, high spatial details and rapid camera scene changes. In this case, the mid-range quality level switches are perceivable to our test subjects. Inspecting the graph shows that the occurrence of quality level switches now has a negative effect on the average rating behaviour, independent of the duration of the switch. Every time a switch is made between high and mid-quality, the average quality rating drops. This indicates that the number of switches throughout the sequence has a bigger effect on quality perception compared to the duration of these switches. This again shows that quality perception is highly content dependent and complies with the research findings from Robinson et al. [25]. In [32], the authors also found that in case of a high action sequence, users have different quality expectations and requirements over extended periods of time which could be attributed to content desirability [36].

\section{Scenario 3: Influence of multi-range bitrate switches}

In the first scenario (Section V-A), we found that switching gradually or instantaneously between high and mid-quality levels of the video has no influence on quality ratings. The latter is also the case for most of the video sequences in our second streaming scenario containing frequent bitrate switches. In this scenario, we investigate the influence of multi-range bitrate switches by considering instant and gradual quality level switches covering the whole range of quality levels as depicted in Fig. 7.
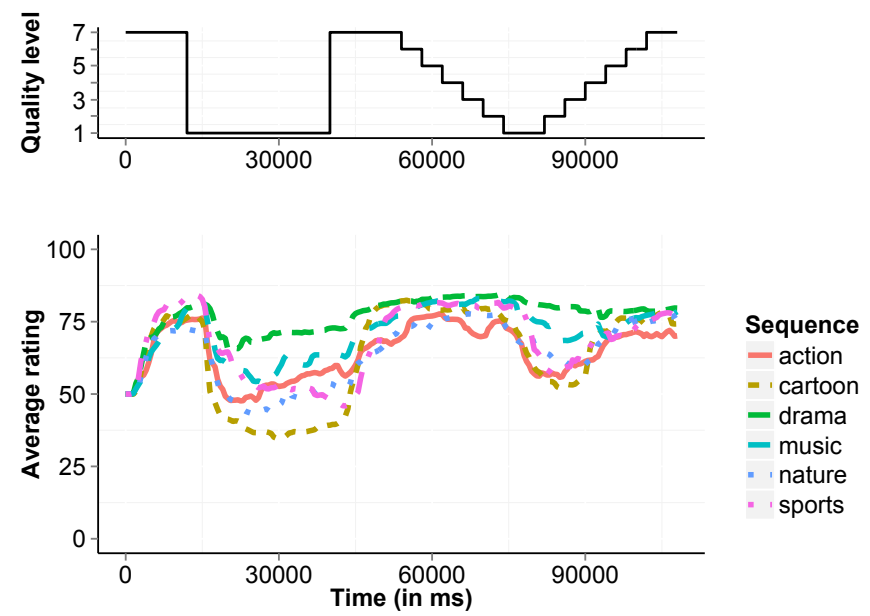

Fig. 7. Overview of the quality switches during video playback in order to assess the influence of multi-range bitrate switches; and corresponding average quality ratings per video sequence.

From the lower part of Fig. 7, it can be seen that the average ratings for all of the sequences exhibit a similar behaviour. Overall, switching between the highest and lowest quality levels instantaneously results in lower average quality ratings compared to gradually downgrading segment quality. On average, the lowest quality level (level 1) is rated 30\% 
better quality in case of gradual quality level switches compared to an instantaneous switch. This corresponds to the research findings in [7] and [21]. As such, gradual bitrate switches are indeed preferred over large multi-range switches since they have a smaller impact on quality perception [23], [24]. Remark, however, that this finding is in our case only valid when switching between the highest and lowest quality levels of the video. This behaviour was not observed when switching between high and mid-quality levels as discussed in the previous scenarios.

Likewise the other scenarios, quality ratings and rating behaviour are impacted by content type. Gradual quality level switches are less visible in low motion sequences.

\section{CONCLUSION}

In this article, we assessed the influence of quality fluctuations during HTTP Adaptive Streaming to mobile devices. In this work, we went beyond existing state-of-the-art subjective studies and recommended assessment methodologies by conducting novel experiments in more ecologically valid environments using long duration video content. As such, we extended the scope of existing subjective studies. Our experiments were conducted on iPad devices in subjects' own home environment. Furthermore, we also included different video content types.

Based on different simulated HAS scenarios, we assessed the influence of bitrate fluctuations and video stallings on quality perception. In this respect, we also considered the influence of switching frequency and switching range. Using continuous quality evaluation we were able to track end-users' behaviour in case of changing video quality.

We found that test subjects are able to track the simulated quality fluctuations. Corresponding to earlier research findings, our results show that, in general, video stallings should be avoided at all times and that gradual quality changes have a smaller influence on quality perception compared to instantaneous switches.

In this study, we found significant influences of video content and the range of quality switches on the average rating behaviour. In our case, quality level switches are only perceived in high motion sequences or in case quality level switches occur between highest and lowest quality of the video. Limited noticeable differences were observed between high and mid-quality switches during video playback.

The results of this study can be used to implement more intelligent adaptation strategies in the HAS clients in order to ensure and maintain QoE towards the end-users at all times. In this respect, as part of the adaptation strategies, both video stallings and high multi-range quality switches should be avoided.

Concerning conducting our experiments in more ecologically valid testing environments, we found that our results deviate less from experiments in controlled environments compared to some of our earlier research on the influence of assessment environment on quality perception. However, as we relied on continuous quality evaluation, test subjects remain concentrated on actively evaluating video quality throughout the entire experiment which hampers an immersive experience.

\section{ACKNOWLEDGMENT}

The research activities described in this paper were funded by Ghent University, iMinds and the Institute for the Promotion of Innovation by Science and Technology in Flanders (IWT). The work is performed as part of the iMinds VFORCE (Video 4K Composition and Efficient streaming) project (under IWT grant agreement no. 130655).

\section{REFERENCES}

[1] Cisco, "Cisco Visual Networking Index: Global Mobile Data Traffic Forecast Update, 20112016," White Paper, February 2012.

[2] Ooyala, "Q2 2013 Video Index," Report, September 2013.

[3] ITU-T Recommendation P.10/G.100 Amd 2, "Vocabulary for performance and quality of service," International Telecommunication Union (ITU), 2008.

[4] DSL Forum Technical Report TR-126, "Triple-play Services Quality of Experience (QoE) requirements," DSL Forum, 2006.

[5] T. Stockhammer, "Dynamic Adaptive Streaming over HTTP - Standards and Design Principles," in Proceedings of the second annual ACM conference on Multimedia systems, ser. MMSys '11. ACM, 2011, pp. 133-144.

[6] N. Staelens, S. Moens, W. Van den Broeck, I. Mariën and, B. Vermeulen, P. Lambert, R. Van de Walle, and P. Demeester, "Assessing quality of experience of IPTV and Video on Demand services in real-life environments," IEEE Transactions on Broadcasting, vol. 56, no. 4, pp. 458-466, December 2010.

[7] A. K. Moorthy, L. K. Choi, A. C. Bovik, and G. de Veciana, "Video Quality Assessment on Mobile Devices: Subjective, Behavioral and Objective Studies," IEEE Journal of Selected Topics in Signal Processing, vol. 6, no. 6, pp. 652-671, 2012.

[8] T. Hossfeld, S. Egger, R. Schatz, M. Fiedler, K. Masuch, and C. Lorentzen, "Initial Delay vs. Interruptions: Between the Devil and the Deep Blue Sea," in Fourth International Workshop on Quality of Multimedia Experience (QoMEX), 2012, pp. 1-6.

[9] ITU-T Recommendation P.910, "Subjective video quality assessment methods for multimedia applications," International Telecommunication Union (ITU), 1999.

[10] ITU-R Recommendation BT.500-13, "Methodology for the subjective assessment of the quality of television pictures," International Telecommunication Union (ITU), 2012.

[11] P. Fröhlich, S. Egger, R. Schatz, M. Muhlegger, K. Masuch, and B. Gardlo, "QoE in 10 Seconds: Are Short Video Clip Lengths Sufficient for Quality of Experience Assessment?" in Fourth International Workshop on Quality of Multimedia Experience (QoMEX), 2012, pp. 242-247.

[12] C. Liu, I. Bouazizi, and M. Gabbouj, "Parallel Adaptive HTTP Media Streaming," in International Conference on Computer Communications and Networks (ICCCN), Aug. 2011, pp. 1-6.

[13] T. Lohmar, T. Einarsson, P. Frojdh, F. Gabin, and M. Kampmann, "Dynamic Adaptive HTTP Streaming of Live Content," in IEEE International Symposium on a World of Wireless, Mobile and Multimedia Networks (WoWMoM), 2011, pp. 1-8.

[14] J. Famaey, S. Latré, N. Bouten, W. Van de Meerssche, B. De Vleeschauwer, W. Van Leekwijck, and F. De Turck, "On the Merits of SVC-Based HTTP Adaptive Streaming," in IFIP/IEEE International Symposium on Integrated Network Management (IM), 2013.

[15] M. Claeys, S. Latré, J. Famaey, T. Wu, W. Van Leekwijck, and F. De Turck, "Design of a Q-Learning-based Client Quality Selection Algorithm for HTTP Adaptive Video Streaming," in Workshop on Adaptive and Learning Agents (ALA), 2013.

[16] V. Menkovski and A. Liotta, "Intelligent control for adaptive video streaming," in IEEE International Conference on Consumer Electronics (ICCE), 2013, pp. 127-128.

[17] ISO/IEC DIS 23009-1, "Information Technology - Dynamic Adaptive Streaming Over HTTP (DASH) - Part 1: Media Presentation Description and Segment Formats," ISO/IEC, August 2011.

[18] Y. Liao, A. Younkin, J. Foerster, and P. Corriveau, "Achieving High QoE Across the Compute Continuum: How Compression, Content, and Devices Interact," Seventh International Workshop on Video Processing and Quality Metrics for Consumer Electronics (VPQM-13), January 2013. 
[19] A. Catellier, M. Pinson, W. Ingram, and A. Webster, "Impact of Mobile Devices and Usage Location on Perceived Multimedia Quality," in Fourth International Workshop on Quality of Multimedia Experience (QoMEX), 2012, pp. 39-44.

[20] N. Staelens, S. Moens, W. Van den Broeck, I. Mariën, B. Vermeulen, P. Lambert, R. Van De Walle, and P. Demeester, "Assessing the Perceptual Influence of H.264/SVC Signal-to-Noise Ratio and Temporal Scalability on Full Length Movies," in International Workshop on Quality of Multimedia Experience (QoMEX), 2009, pp. 29-34.

[21] R. K. P. Mok, X. Luo, E. W. W. Chan, and R. K. C. Chang, "Qdash: a QoE-aware DASH System," in Proceedings of the 3rd Multimedia Systems Conference, ser. MMSys '12. ACM, 2012, pp. 11-22.

[22] K. Boff, L. Kaufman, and J. Thomas, Handbook of Perception and Human Performance: Sensory Processes and Perception. Wiley, 1986.

[23] P. Reichl, S. Egger, R. Schatz, and A. D'Alconzo, "The Logarithmic Nature of QoE and the Role of the Weber-Fechner Law in QoE Assessment," in IEEE International Conference on Communications (ICC), 2010, pp. 1-5

[24] C.-N. Chen, C.-Y. Chu, S.-L. Yeh, H.-H. Chu, and P. Huang, "Modeling the QoE of Rate Changes in SKYPE/SILK VoIP Calls," in Proceedings of the 20th ACM international conference on Multimedia, ser. MM '12. New York, NY, USA: ACM, 2012, pp. 119-128.

[25] D. C. Robinson, Y. Jutras, and V. Craciun, "Subjective Video Quality Assessment of HTTP Adaptive Streaming Technologies," Bell Labs Technical Journal, vol. 16, no. 4, 2012.

[26] A. Floris, L. Atzori, G. Ginesu, and D. D. Giusto, "QoE Assessment of Multimedia Video Consumption on Tablet Devices," in IEEE Globecom Workshop: Quality of Experience of Multimedia Communications, 2012, pp. 1329-1334.

[27] L. Atzori, A. Floris, G. Ginesu, and D. D. Giusto, "Quality Perception when Streaming Video on Tablet Devices," Journal of Visual Communication and Image Representation, 2013.

[28] R. K. P. Mok, E. W. W. Chan, and R. K. C. Chang, "Measuring the Quality of Experience of HTTP Video Streaming," in IFIP/IEEE International Symposium on Integrated Network Management (IM), 2011, pp. 485-492.

[29] F. Dobrian, V. Sekar, A. Awan, I. Stoica, D. Joseph, A. Ganjam, J. Zhan, and H. Zhang, "Understanding the impact of video quality on user engagement," in Proceedings of the ACM SIGCOMM 2011 conference, ser. SIGCOMM '11. New York, NY, USA: ACM, 2011, pp. 362-373.

[30] A. ParandehGheibi, M. Médard, A. Ozdaglar, and S. Shakkottai, "Avoiding Interruptions - A QoE Reliability Function for Streaming Media Applications," IEEE Journal on Selected Areas in Communications, vol. 29, no. 5, pp. 1064-1074, 2011.

[31] Y. Xu, E. Altman, R. El-Azouzi, M. Haddad, S. Elayoubi, and T. Jimenez, "Probabilistic analysis of buffer starvation in markovian queues," in IEEE INFOCOM Proceedings, 2012, pp. 1826-1834.

[32] A. Borowiak and U. Reiter, "Long Duration Audiovisual Content: Impact of Content Type and Impairment Appearance on User Quality Expectations over Time," in Fifth International Workshop on Quality of Multimedia Experience (QoMEX), 2013, pp. 200-205.

[33] S. Jumisko-Pyykkö and M. M. Hannuksela, "Does Context Matter in Quality Evaluation of Mobile Television?" in Proceedings of the 10th International Conference on Human Computer Interaction with Mobile Devices and Services. New York, NY, USA: ACM, 2008, pp. 63-72.

[34] M. C. Q. Farias and S. K. Mitra, "A Methodology for Designing No-Reference Video Quality Metrics," Fourth International Workshop on Video Processing and Quality Metrics for Consumer Electronics (VPQM-09), January 2009.

[35] M. H. Pinson, K. S. Boyd, J. Hooker, and K. Muntean, "How to Choose Video Sequences for Video Quality Assessment," Seventh International Workshop on Video Processing and Quality Metrics for Consumer Electronics (VPQM-13), January 2013.

[36] P. Kortum and M. Sullivan, "The Effect of Content Desirability on Subjective Video Quality Ratings," Human Factors: The Journal of the Human Factors and Ergonomics Society, vol. 52, no. 1, pp. 105-118, 2010.

[37] P. Corriveau, Video Quality Testing, ser. Digital Video Image Quality and Perceptual Coding. CRC Press, 2006, ch. 4, pp. 125-153.

[38] A. Ostaszewska and R. Kloda, "Quantifying the amount of spatial and temporal information in video test sequences," in Recent Advances in Mechatronics. Springer Berlin Heidelberg, 2007, pp. 11-15.

[39] Technical Note TN2224, "Best Practices for Creating and Deploying HTTP Live Streaming Media for the iPhone and iPad," Apple Technical Note, 2012. [Online]. Available: https://developer.apple.com/library/ios/technotes/tn2224/_index.html
[40] C. Liu, I. Bouazizi, and M. Gabbouj, "Segment Duration for Rate Adaptation of Adaptive HTTP Streaming," in IEEE International Conference on Multimedia and Expo (ICME), 2011, pp. 1-4.

[41] M. H. Pinson and S. Wolf, "Comparing Subjective Video Quality Testing Methodologies," SPIE Visual Communications and Image Processing Conference, vol. 5150, pp. 573-582, 2003.

[42] M. Matulin and Š. Mrvelj, "State-of-the-Practice in Evaluation of Quality of Experience in Real-Life Environments," Promet - Traffic \& Transportation, vol. 25, no. 3, pp. 255-263, 2013.

[43] W. Van den Broeck, A. Jacobs, and N. Staelens, "Integrating the Everyday-Life Context in Subjective Video Quality Experiments," in Fourth International Workshop on Quality of Multimedia Experience (QoMEX), July 2012.

[44] H. de Ridder and R. Hamberg, "Continuous Assessment of Image Quality,” SMPTE Journal, vol. 106, no. 2, pp. 123-128, 1997.

[45] S. Winkler and R. Campos, "Video Quality Evaluation for Internet Streaming Applications," Proc. SPIE, vol. 5007, pp. 104-115, 2003.

[46] N. Suresh, N. Jayant, and O. Yang, "Mean Time Between Failures: A Subjectively Meaningful Quality Metric for Consumer Video," Second International Workshop on Video Processing and Quality Metrics for Consumer Electronics (VPQM-06), January 2006.

[47] K. Seshadrinathan and A. Bovik, "Temporal Hysteresis Model of Time Varying Subjective Video Quality," in IEEE International Conference on Acoustics, Speech and Signal Processing (ICASSP), 2011, pp. 11531156.

[48] M. Seufert, M. Slanina, S. Egger, and M. Kottkamp, "To Pool or not to Pool: A Comparison of Temporal Pooling Methods for HTTP Adaptive Video Streaming," in Fifth International Workshop on Quality of Multimedia Experience (QoMEX), 2013, pp. 52-57.

[49] M. Pinson, M. Barkowsky, and P. Le Callet, "Selecting Scenes for 2D and 3D Subjective Video Quality Tests," EURASIP Journal on Image and Video Processing, vol. 2013, no. 50, pp. 1-12, 2013.

[50] Q. Huynh-Thu, M.-N. Garcia, F. Speranza, P. Corriveau, and A. Raake, "Study of Rating Scales for Subjective Quality Assessment of HighDefinition Video," IEEE Transactions on Broadcasting, vol. 57, no. 1, pp. 1-14, march 2011.

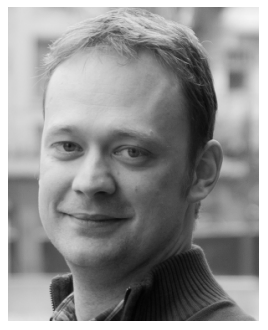

Nicolas Staelens obtained his Master's degree in Computer Science at Ghent University (Belgium, 2004). He started his career as an $R \& D$ engineer at Televic Healthcare, a Belgian company that develops, designs and manufactures high-end network systems and software applications for the healthcare market. In 2006, he joined the Internet Based Communication Networks and Services (IBCN) group at Ghent University where he received a $\mathrm{Ph} . \mathrm{D}$. degree in Computer Science Engineering in February 2013. The topic of his dissertation was "Objective and Subjective Quality Assessment of Video Distributed over IP-based Networks". Currently, he is still working at the same university where his main research focuses on assessing the influence of network impairments on perceived audiovisual quality. As of 2007 , he is also actively participating within the Video Quality Experts Group (VQEG) and is currently co-chair of the Tools and Subjective Labs support group and the JEG-Hybrid project.

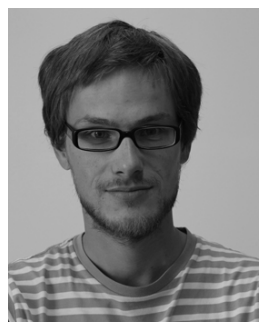

Jonas De Meulenaere Jonas studied Communication sciences at Ghent University. In 2010 he graduated with a master's thesis on the contextualized use of Mobile Internet. From 2011 on, Jonas works as a researcher at SMIT on projects related to digital media in the home and video quality, including the iMinds projects 3DTV 2.0, OMUS and MISTRAL. 


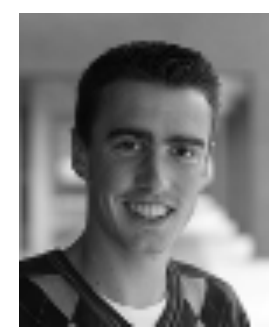

Maxim Claeys obtained a masters degree in computer science from Ghent University, Belgium, in June 2012. In August 2012, he joined the Department of Information Technology at Ghent University, where he is active as a Ph.D. student. His main research interests are the application of autonomic network management approaches in multimedia delivery. The focus of this research is mainly on the end-to-end Quality of Experience optimization, ranging from the design of autonomous clients to intelligent in-network decision taking.

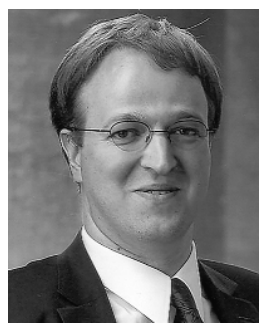

Rik Van de Walle received his M.Sc. and $\mathrm{PhD}$ degrees in Engineering from Ghent University, Belgium in 1994 and 1998 respectively. After a visiting scholarship at the University of Arizona (Tucson, USA), he returned to Ghent University, where he became professor of multimedia systems and applications, and head of the Multimedia Lab. His current research interests include multimedia content delivery, presentation and archiving, coding and description of multimedia data, content adaptation, and interactive (mobile) multimedia applications.

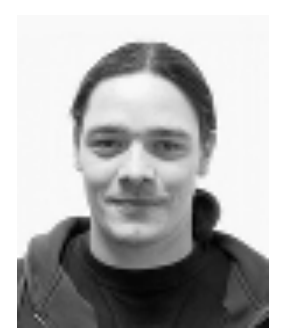

Glenn Van Wallendael obtained the M.Sc. degree in Applied Engineering from the University College of Antwerp, Belgium, in 2006 and the M.Sc. degree in Engineering from Ghent University, Belgium in 2008. Afterwards, he worked towards a Ph.D. at Multimedia Lab, Ghent University, with the financial support of the Agency for Innovation by Science and Technology (IWT). Currently, he continues working in the same group as a post-doctoral researcher. His main topics of interest are video compression including scalable video compression and transcoding.

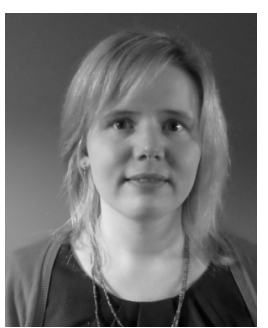

Wendy Van den Broeck Prof. Dr. Wendy van den Broeck is senior researcher at iMinds-SMIT, Brussels, Belgium. She is active in the user Empowerment research unit and conducted research in different projects concerning the domestication of new media technologies in a home context. Her recent projects are related to multi-screen user practices, the use and quality perception of video streaming and research related to iDTV, 3D-TV, second screen applications and HDTV/4K TV. Her teaching activities focus on social research methodologies. She conducted a PhD research on the domestication of iDTV in Flanders, entitled: "From analogue to digital: the silent (r)evolution? A qualitative study on the domestication of interactive digital television in Flanders. Her academic background is a master in communication sciences (VUB-2002), a postgraduate diploma in management and economics (VUB-Solvay-2003), a teaching degree (VUB-2003) and a PhD in communication sciences (VUBDecember 2010).

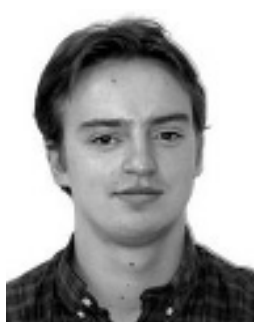

Jan De Cock obtained the M.S. and Ph.D. degrees in Engineering from Ghent University, Belgium, in 2004 and 2009, respectively. Since 2004 he has been working at Multimedia Lab, Ghent University, iMinds. In 2010, he obtained a postdoctoral research fellowship from the Flemish Agency for Innovation by Science and Technology (IWT) and in 2012, a postdoctoral research fellowship from the Research Foundation Flanders (FWO). His research interests include high-efficiency video coding and transcoding, scalable video coding, and multimedia

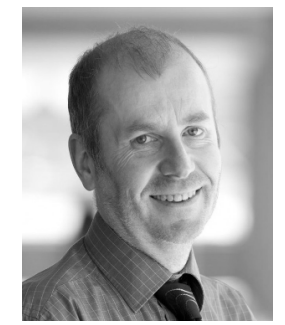

Piet Demeester is professor in the faculty of Engineering at Ghent University. $\mathrm{He}$ is head of the research group "Internet Based Communication Networks and Services" (IBCN) that is part of the Department of Information Technology (INTEC) of Ghent University. He is also leading the Future Internet (Networks, Media and Service) Department of the Interdisciplinary Institute for Broadband Technology (iMinds). He is Fellow of the IEEE. After finishing a $\mathrm{PhD}$ on Metal Organic Vapor Phase Epitaxy for photonic devices in 1988, he established a research group in this area working on different material systems (AlGaAs, InGaAsP, GaN). This research was successfully transferred to IMEC in 2002 and resulted in $12 \mathrm{PhDs}$ and 300 publications in international journals and conference proceedings. In 1992 he started research on communication networks and established the IBCN research group. The group is focusing on several advanced research topics: Network Modeling, Design \& Evaluation; Mobile \& Wireless Networking; High Performance Multimedia Processing; Autonomic Computing \& Networking; Service Engineering; Content \& Search Management and Data Analysis \& Machine Learning. The research of IBCN resulted in about $50 \mathrm{PhD}$ 's, 1250 publications in international journals and conference proceedings, 30 international awards and 4 spin-off companies.

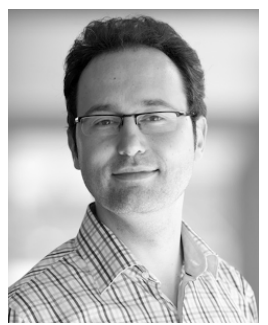

Filip De Turck received his M.Sc. degree in Electronic Engineering from the Ghent University, Belgium, in June 1997. In May 2002, he obtained the Ph.D. degree in Electronic Engineering from the same university. During his Ph.D. research he was funded by the F.W.O.-V., the Fund for Scientific Research Flanders. From October 2002 until September 2008, he was a post-doctoral fellow of the F.W.O.-V. and part time professor, affiliated with the Department of Information Technology of the Ghent University. At the moment, he is a full-time professor affiliated with the Department of Information Technology of the Ghent University and iMinds (Interdisciplinary Institute of Broadband Technology Flanders) in the area of telecommunication and software engineering. Filip De Turck is author or co-author of approximately 250 papers published in international journals or in the proceedings of international conferences. His main research interests include scalable software architectures for telecommunication network and service management, performance evaluation and design of new telecommunication and eHealth services. 